\title{
Critical Motion Sequences for Monocular Self-Calibration and Uncalibrated Euclidean Reconstruction
}

\author{
Peter Sturm \\ GRAVIR-IMAG \& INRIA Rhône-Alpes ${ }^{\star}$ \\ 655, Avenue de l'Europe, 38330 Montbonnot, France \\ Peter.Sturmeinrialpes.fr
}

\begin{abstract}
In this paper, sequences of camera motions that lead to inherent ambiguities in uncalibrated Euclidean reconstruction or self-calibration are studied. Our main contribution is a complete, detailed classification of these critical motion sequences (CMS). The practically important classes are identified and their degrees of ambiguity are derived. We also discuss some practical issues, especially concerning the reduction of the ambiguity of a reconstruction.
\end{abstract}

\section{Introduction}

Uncalibrated Vision and Self-Calibration. One of the major goals of computer vision is the recovery of spatial information about the environment. Classical approaches assume that the cameras are calibrated beforehand, but a great interest in uncalibrated vision and on-line calibration has arisen during the last couple of years. A key result is that even with completely uncalibrated cameras, spatial information - projective structure - can be obtained: the scene can be reconstructed up to an unknown projective transformation [7, 9]. Furthermore, a moving camera can self-calibrate, i.e. the calibration parameters can be estimated solely from feature correspondences between several images [16 $]^{2}$. This allows the projective ambiguity in the reconstruction to be reduced to a Euclidean one (up to a similarity transformation), and we speak of monocular uncalibrated Euclidean reconstruction.

Critical Motion Sequences. It is known that several types of camera motion prevent self-calibration, i.e. the calibration parameters can not be determined uniquely. Accordingly, Euclidean structure can not be obtained, although reconstruction at some level between projective and Euclidean is generally possible. For example, from pure translations, even in different directions, affi ne structure can be obtained [17], while general planar motions of the camera allow a Euclidean reconstruction up to a scale ambiguity in one direction [2, 25].

These ambiguities are inherent, i.e. they can not be resolved by any algorithm without additional knowledge. Sequences of camera motions that imply such ambiguities will be referred to as critical motion sequences (CMS). By "sequences" we mean that not only the motion between two frames, but that over the complete sequence of frames, is critical. Another type of inherent ambiguity in structure recovery is caused by critical surfaces: if all observed points lie on a special surface (certain ruled quadrics) and the cameras have a special position with respect to that surface, then the structure can not be recovered uniquely $[13,14]$. Contrary to critical surfaces, critical motion sequences imply ambiguities for any scene!

Beside these fundamental ambiguities, others arising from numerical instability due to noisy measurements have been studied, especially for two calibrated cameras and affi ne camera models [1,22]. Numerical instability can also be caused by near-critical motion sequences, but an analytical study of these effects is beyond the scope of this paper.

Critical motion sequences have already become established in practical works on self-calibration through the development of algorithms specially designed for certain types of CMS [2, 11, 17, 25]. However, if applied to other motion sequences, they will fail. Conversely, algorithms developed for general camera motion [10, 12, 15, 18, 23] will, if

\footnotetext{
* This work has been done in the context of the Movi project which belongs to CNRS, INPG, INRIA and UJF.

${ }^{2}$ Approaches that assume special camera motions, are sometimes included in self-calibration (e.g. [2, 3, 5, 6, 11]). However, we prefer to call this calibration from motion constraints. We also distinguish bundle adjustment with self-calibration [20], which accurately calibrates the cameras while simultaneously reconstructing the scene. However, an initial approximate camera calibration and/or known control points are needed.
} 
applied to critical sequences, hopefully fi nd one of the ambiguous solutions, but this will generally not be the correct one.

The problem of critical motion sequences is important, since many image sequences used for object modeling are indeed critical, as will become clear in sections 4 and 5. In spite of their importance, we are not aware of any complete investigation of CMS in literature. A study in this direction is reported in [26], but the problem is only considered for 3 frames and is linked to a particular self-calibration algorithm.

In this paper, we derive a complete characterization of critical motion sequences, which is independent of the number of frames and of the algorithm used. We also show which of the sequences permit at least affi ne scene reconstruction or camera self-calibration.

Basic Idea. Now we sketch the basic idea behind the derivation of the critical motion sequences. Euclidean reconstruction is equivalent to the determination of the absolute conic $\Omega$ [8]. This can only be based upon the special properties which distinguish it from all other conics in 3-space. The main property, and usually the only one used in existing algorithms, is that the projection of $\Omega$ is invariant under camera motions provided the intrinsic parameters do not change. Its image $\omega$ can thus be determined as the "fi xed conic of a sequence" [2]. Furthermore, $\Omega$ is a proper virtual conic, and for perfect perspective projection, its images must also be proper virtual conics. Besides these properties (or equivalent ones) there is no means to determine $\Omega$ from monocular uncalibrated image sequences.

Hence, the problem of monocular uncalibrated Euclidean reconstruction fails to have a unique solution exactly when there is at least one other conic besides $\Omega$ with the same properties, i.e. a proper virtual conic $\Omega^{\prime}$ that is projected onto some proper virtual conic $\omega^{\prime}$ in all frames of the sequence.

Structure of the Paper. In section 2 we provide the theoretical background of our approach. Basic defi nitions are settled in section 3 and used in section 4 to derive the critical motion sequences. A taxonomy of the CMS and some of their properties are presented in section 5. Practical issues are discussed and conclusions are drawn in sections 6 and 7.

\section{Background}

The defi nitions in this section are mainly taken from [4] and [19]. Some of the results for general quadrics are presented only for central conics.

Notation. We refer to the plane at infinity as the ideal plane and denote it by $\Pi_{\infty} . \mathcal{P}^{n}$ is the $n$-dimensional projective space and $\sim$ means equality up to a scalar factor. We use the abbreviation $P V C$ for proper virtual conics (see below).

Pinhole Camera Model. In this paper, we use the projective pinhole camera model. The projection can be represented by a $3 \times 4$ projection matrix $\mathbf{P}$. We consider only the case of perfect perspective projection, i.e. the projection center does not lie on $\Pi_{\infty}$. With regard to physical cameras, the projection matrix can be decomposed into a calibration matrix $\mathbf{A}$ and a pose matrix $\mathbf{T}$. The pose matrix represents the position and orientation of the camera in some absolute coordinate frame. The calibration matrix describes the invertible affi ne transformation from the canonical projection to pixel coordinates. For the pinhole model, the calibration matrix is determined by 5 intrinsic parameters: focal length, measured in horizontal and vertical pixels, two coordinates of the principal point, and skew angle between the pixel axes.

Relative Rotation. The relative rotation between two frames whose orientation is given by rotation matrices $\mathbf{R}_{\mathbf{1}}$ and $\mathbf{R}_{\mathbf{2}}$, is represented by the rotation matrix $\mathbf{R}=\mathbf{R}_{\mathbf{1}}^{\mathbf{T}} \mathbf{R}_{\mathbf{2}}$.

Quadrics and Conics. A quadric in $\mathcal{P}^{n}$ is a set of points satisfying a homogeneous quadratic equation. Each quadric can be represented by a symmetric $(n+1) \times(n+1)$ matrix. A virtual quadric is a quadric with no real point and a proper quadric is a quadric whose matrix has a non-zero determinant. Conics are planar quadrics; we will not distinguish between a conic and its matrix. A conic in $\mathcal{P}^{3}$, or $3 D$ conic, is defi ned by its supporting plane and the conic's equation in that plane. All proper virtual conics $(P V C)$ are central [4] and hence can be transformed to Euclidean normal form by a Euclidean transformation (principal axis transformation). The Euclidean normal form is a diagonal matrix of the conic's eigenvalues. If all three eigenvalues are distinct, then the conic has exactly two symmetry lines and is an ellipse. The symmetry lines pass through the conic's center and are mutually orthogonal. If the eigenvalues are not all distinct, the conic is a circle and all lines through the center are symmetry lines.

Symmetry Planes of $3 D$ Conics. We defi ne the symmetry planes of a proper $3 D$ conic $\Phi$ as the planes reflecting $\Phi$ onto itself. The symmetry planes of $\Phi$ are exactly its supporting plane $\Pi$ and the planes perpendicular to $\Pi$ that contain a symmetry line of $\Phi$. 
Cones. By cones we mean rank-3 quadrics in $\mathcal{P}^{3}$ with vertex not on $\Pi_{\infty}$. A cone is uniquely defi ned by its vertex and any (conic) section by a plane not containing the vertex. Cones are used in this paper through the notion of the projection cone of a 3D conic, i.e. the cone traced out by the projection rays of the perspective projection of the conic. The Euclidean normal form of a cone is a diagonal matrix $\operatorname{diag}\left(\lambda_{1}, \lambda_{2}, \lambda_{3}, 0\right)$, with non-zero $\lambda_{i}$. If the $\lambda_{i}$ are all distinct then the cone is an elliptic cone and has 3 mutually orthogonal axes. If exactly two of the $\lambda_{i}$ are equal, the cone is circular. For an absolute cone, all three $\lambda_{i}$ are equal.

Absolute Quadric and Absolute Conic. The absolute quadric of $\mathcal{P}^{n}$ is defi ned by the equations $x_{1}^{2}+\cdots+x_{n}^{2}=$ $x_{n+1}=0$. The absolute conic $\Omega$ is the absolute quadric of $\mathcal{P}^{3} . \Omega$ is a proper virtual conic in the ideal plane whose position uniquely defi nes the Euclidean structure of 3-space. The calibration of a camera is equivalent to determining the image $\omega$ of $\Omega$, respectively its dual $\omega^{*}[16,8]$. From the relation $\omega^{*} \sim \mathbf{A A}^{\mathbf{T}}$, the calibration matrix $A$ can be uniquely recovered by Cholesky decomposition.

\section{Basic Definitions}

We defi ne a motion sequence $S$ of $m$ camera positions as $S=\left(\mathbf{R}_{\mathbf{i}}, \mathbf{t}_{\mathbf{i}}\right)_{\mathbf{i}=\mathbf{1}}^{\mathbf{m}}$, where $\left(\mathbf{R}_{\mathbf{i}}, \mathbf{t}_{\mathbf{i}}\right)$ are the rotational and translational components of the $i$ th camera pose.

\subsection{Critical Motion Sequences}

We fi rst note that the question of whether a given motion sequence is critical is independent of the cameras intrinsic parameters: a conic has the same image in a set of views taken by the same camera, exactly if it has the same image in the corresponding canonical projections ${ }^{3}$. It is thus suffi cient to consider only the pose of the camera.

Definition 1. Let $S$ be a motion sequence and $\mathbf{P}_{\mathbf{i}}$ the canonical projection for the $i$ th frame. Let $\mathbf{P}_{\mathbf{i}}(\Phi)$ be the image of the $3 \mathrm{D}$ conic $\Phi$.

The motion sequence $S$ is critical if there exists a proper virtual conic $\Phi$, distinct from $\Omega$, that projects to the same proper virtual conic $\phi$ in all frames of $S: \phi \sim \mathbf{P}_{\mathbf{i}}(\Phi)$ for $i=1, \ldots, m$.

Such PVC $\Phi$ will be referred to as potential absolute conics and we say that the motion sequence $S$ is critical with respect to $\Phi$.

It follows that Euclidean reconstruction from an uncalibrated monocular image sequence is ambiguous exactly when the underlying camera motion is a critical motion sequence (cf. the basic idea described in section 1).

\subsection{Affine Reconstruction}

In view of the stratifi cation of reconstruction [8] the question naturally arises, which motion sequences allow at least an affi ne reconstruction of the scene. Affi ne reconstruction is equivalent to the determination of the ideal plane $\Pi_{6}$ in a projective reconstruction. $\Pi_{\infty}$ is uniquely characterized as the supporting plane of the absolute conic ${ }^{4}$. Hence, if for a given motion sequence there exist potential absolute conics with different supporting planes, then the ideal plane can not be uniquely identifi ed and affi ne reconstruction is not possible:

Definition 2. A motion sequence is critical for affine reconstruction if it is critical with respect to a proper virtual conic $\Phi$, which is not on the ideal plane.

\subsection{Self-Calibration}

While Euclidean reconstruction implies the (self-) calibration of the camera, the inverse is not always true: a camera which rotates about its projection center can in general self-calibrate [11], but because of the missing base-line, no reconstruction is possible. The fact that (self-) calibration is equivalent to the determination of the image of the absolute conic, leads to the following defi nition:

\footnotetext{
${ }^{3}$ There is an invertible affine transformation between the canonical projection and the image plane (see section 2).

${ }^{4}$ In [18] the constraint that the infinite homography has eigenvalues of the same modulus is used for affine reconstruction. We show in [21] that this is a necessary but insufficient constraint.
} 
Definition 3. A motion sequence $S$ is critical for self-calibration if it is critical with respect to a PVC, whose image $\phi$ is different from that of the absolute conic.

In the case of a rotating camera, there indeed exist an infi nity of potential absolute conics, but they all lie on the projection cone of the absolute conic. This is why the images of all potential absolute conics are identical and equal to the image of the absolute conic, which means that self-calibration is possible!

\section{Derivation of the CMS}

In this section, the critical motion sequences are derived, based on the previous defi nitions. We divide the derivations into two parts, for potential absolute conics which lie / do not lie on $\Pi_{\infty}$. The results are summarized in section 5 . For more details and formal proofs refer to [21].

\subsection{Potential Absolute Conics on $\Pi_{\infty}$}

\subsubsection{Constraint on Relative Rotations}

Let $\Phi$ be a PVC on the ideal plane. Its (canonical) perspective projection $\phi$ by a projection matrix $\mathbf{P}=(\mathbf{R} \mid \mathbf{t})$ is given by $\phi \sim \mathbf{R} \Phi \mathbf{R}^{\mathbf{T}}$. Note that the projection of $\Phi$ depends only on the orientation of the camera, not on its position.

Consider two frames with orientations $\mathbf{R}_{\mathbf{1}}$ and $\mathbf{R}_{\mathbf{2}}$. The projection of $\Phi$ is the same in both frames if $\mathbf{R}_{\mathbf{1}} \Phi \mathbf{R}_{\mathbf{1}}^{\mathbf{T}} \sim$ $\mathbf{R}_{\mathbf{2}} \Phi \mathbf{R}_{\mathbf{2}}^{\mathbf{T}}$. Since the determinants of rotation matrices are 1, we obtain the condition $\mathbf{R}_{\mathbf{1}} \Phi \mathbf{R}_{1}^{\mathbf{T}}=\mathbf{R}_{\mathbf{2}} \Phi \mathbf{R}_{\mathbf{2}}^{\mathbf{T}}$. Introducing the relative rotation $\mathbf{R}$ between the two frames, the condition that $\Phi$ has the same projection in both frames becomes

$$
\mathbf{R} \Phi=\Phi \mathbf{R} .
$$

Let $\mathbf{x}$ be an eigenvector of $\Phi$ with eigenvalue $\lambda$. From Equation (1) it follows that $\Phi \mathbf{R x}=\mathbf{R} \Phi \mathbf{x}=\lambda \mathbf{R x}$, i.e. $\mathbf{R x}$ is also an eigenvector of $\Phi$ with eigenvalue $\lambda$. This is valid for all eigenvectors $\mathbf{x}$ of $\Phi$ and it follows that $\mathbf{R}$ conserves the eigenspaces of $\Phi$. Hence, for a PVC $\Phi$ on the ideal plane to have the same image in all frames of a sequence, all relative rotations between pairs of frames must conserve the eigenspaces of $\Phi$. This is also a suffi cient condition [21].

\subsubsection{Developing the Constraint}

We develop the condition on the relative rotations with respect to the various possible forms of $\Phi$. The criticality of a motion sequence does not depend on the absolute coordinate frame in which the poses are expressed, so we can take $\Phi$ to have Euclidean normal form. Since $\Phi$ is proper virtual, there are exactly 3 different cases, differing in the multiplicity of the eigenvalues of $\Phi$ :

Case 1. One Triple Eigenvalue. The only conic on $\Pi_{\infty}$ with a triple eigenvalue is the absolute conic, so the sequence is not critical.

Case 2. One Double and One Single Eigenvalue. The eigenspace of the double eigenvalue is a plane $\Pi$ and that of the single eigenvalue a line $\mathbf{l}$ perpendicular to $\Pi^{5}$. Since the rotation $\mathbf{R}$ conserves $\Pi$ and $\mathbf{l}$, it must be either the identity transformation, a rotation about $\mathbf{l}$ by an arbitrary angle, or a rotation by $180^{\circ}$ about a line on $\Pi$, incident with $\mathbf{l}$.

Case 3. Three Single Eigenvalues. The eigenspaces are three mutually orthogonal lines. $\mathbf{R}$ is thus the identity transformation or a rotation by $180^{\circ}$ about any of the 3 lines.

Note that the relative rotations $\mathbf{R}$ that arise from Case 3 are also covered by Case 2. The following class covers all motion sequences that are critical with respect to a PVC on $\Pi_{\infty}$ :

CMS-Class 1: Motion sequences for which all relative rotations are either rotations by an arbitrary amount about some line 1 or by $180^{\circ}$ about lines perpendicular to 1 .

If a conic $\Phi$ on $\Pi_{\infty}$ has the same image in all frames of a sequence, this is also true for all conics in the linear family spanned by $\Phi$ and $\Omega$. Hence, there exists a familiy of ambiguous Euclidean reconstructions. Class 1 is rather large but we can identify more specifi c sub-classes:

\footnotetext{
${ }^{5}$ Eigenvectors of symmetric matrices corresponding to different eigenvalues are orthogonal.
} 
CMS-Class 1.1: All relative rotations are arbitrary rotations about some line $\mathbf{l}$.

CMS-Class 1.2: All relative rotations are rotations by $180^{\circ}$ about 3 mutually orthogonal lines.

CMS-Class 1.3: All relative rotations are rotations by $180^{\circ}$ about some line $\mathbf{l}$.

CMS-Class 1.4: There is no relative rotation at all, i.e. the motions are pure translations in arbitrary directions.

A further discussion is given in section 5.

\subsection{Potential Absolute Conics not on $\Pi_{\infty}$}

Conics not on $\Pi_{\infty}$ are rather more diffi cult to deal with, since their projection depends on both the position and orientation of the camera. An algebraic description of the CMS through constraints on the position and orientation of the frames in a sequence is very tedious to derive [26] and it is not at all clear if it is tractable for more than 3 frames. We thus preferred a more intuitive geometrical approach. This does not provide an exact algebraic description but it does yield a geometric description of all types of critical motion sequences, for any number of frames.

\subsubsection{Problem Formulation}

We construct the critical motion sequences generically by solving the following problem:

Problem 4. Let $\Phi$ be a PVC that does not lie on $\Pi_{\infty}$. Let $\phi$ be its projection for an initial camera pose. Determine all poses, for which the projection of $\Phi$ equals $\phi$. Any subset of these forms a CMS. Equivalently, we may determine all rigid transformations mapping the initial pose to poses where the projection of $\Phi$ equals $\phi$.

We transform this into an equivalent, more tractable form:

Problem 5. Let $\Phi$ and $\phi$ be given as in Problem 4. Let $\mathbf{K}$ be the projection cone of $\Phi$ for the initial pose. Determine all rigid transformations mapping $\mathbf{K}$ to cones containing $\Phi$.

\subsubsection{Solving Problem 5}

In order to cover the whole range of possible critical motion sequences, we must treat all different forms of proper virtual conics and also the different possibilities which arise when choosing the initial camera pose. The fact that $\Phi$ is a PVC greatly reduces the range of conics we have to deal with. Furthermore, we can immediately exclude camera positions on the supporting plane of $\Phi$, one of the properties of potential absolute conics being that their images are proper.

We solve Problem 5 through two key observations: (i) Planar reflections, although not orientation preserving, induce transformations on a cone that are setwise equivalent to rigid motions. (ii) The rigid transformations required in Problem 5 are exactly those induced by certain planar reflections of the projection cone $\mathbf{K}$. In concrete terms (see [21] for proofs):

Lemma 6. The reflection of a quadric $\mathbf{K}$ in a hyperplane is a quadric $\mathbf{K}^{\prime}$ which is equal to $\mathbf{K}$ up to a rigid transformation.

Lemma 7. Let $\Phi$ be a PVC that does not lie on $\Pi_{\infty}$. Let $\mathbf{K}_{\mathbf{1}}$ be a cone containing $\Phi$ and $\mathbf{K}_{\mathbf{2}}$ another that equals $\mathbf{K}_{\mathbf{1}}$ up to a rigid transformation. Then $\mathbf{K}_{\mathbf{2}}$ contains $\Phi$ exactly when $\mathbf{K}_{\mathbf{2}}$ can be obtained from $\mathbf{K}_{\mathbf{1}}$ by a combination of reflections in symmetry planes of $\Phi$.

Hence, the combinations of rigid transformations induced by planar reflections of $\mathbf{K}$ in symmetry planes of $\Phi$, are exactly the rigid transformations solving Problem 5 and thus Problem 4. An explicit description of these motions [21] is omitted, due to lack of space. Instead we concentrate on the essential features of the description: the number and locus of the camera positions and corresponding orientations. 


\subsubsection{Camera Positions}

Since $\Phi$ is a proper virtual conic it must be a circle or an ellipse. The symmetry planes of a circle are its supporting plane $\Pi$ and all planes orthogonal to $\Pi$ containing the circle's center. An ellipse has 3 symmetry planes: $\Pi$ and the 2 planes orthogonal to $\Pi$ containing an axis of the ellipse. Consider a point $\mathbf{Q}$ and apply all combinations of planar reflections in symmetry planes of $\Phi$. For an ellipse $\Phi$, the locii of reflected points are the 8 corners of a rectangular parallelepiped. These may even collapse, if $\mathbf{Q}$ lies on one or more symmetry planes. For a circle $\Phi$, the locii are two equally sized circles, whose supporting planes are parallel and equidistant to $\Pi$. We immediately draw the important conclusion that the projection centers in motion sequences that are critical with respect to a PVC not on $\Pi_{\infty}$, lie at most on two parallel and equally sized circles (the corners of a rectangular parallelepiped lie always on such circles)!

\subsubsection{Camera Orientations}

For any cone, rotations about its vertex exist that leave the cone globally invariant. We refer to these as local orientation changes. The number of local orientation changes depends on the cone's Euclidean normal form: arbitrary rotation of an absolute cone about its vertex leave the cone globally unchanged; a circular cone may be rotated by any degree about its main axis or by $180^{\circ}$ by any line perpendicular and incident to the main axis; elliptic cones are left globally invariant by rotations by $180^{\circ}$ about their axes. We count the local orientation changes as follows: $\infty^{3}$ for an absolute cone, $2 \times \infty$ for a circular cone and 4 for an elliptic cone (including the identity transformation). Consider a frame in a motion sequence that is critical with respect to a PVC $\Phi$ not lying on $\Pi_{\infty}$. Let $\mathbf{K}$ be the projection cone of $\Phi$ in the considered frame. Any rigid transformation that leaves $\mathbf{K}$ globally invariant, i.e. any local orientation change, possibly changes $\Phi$, but not its image, because the transformed $\Phi$ still lies on the projection cone $\mathbf{K}$. Dually, changing the orientation of the camera by any local orientation change of $\mathbf{K}$ has no effect on the image of $\Phi$.

\subsubsection{Synthesis}

We synthesize the derivations of the previous subsections by considering camera position and orientation together. All combinations of the following possibilities must be considered: $\mathbf{K}$ is an elliptic, a circular or an absolute cone and $\Phi$ is an elliptic or a circular virtual conic. Let in the following $\Pi$ be the supporting plane of $\Phi$.

Case 4. K: Absolute cone, $\Phi$ : Ellipse. All planar sections of absolute cones are circles, so this case is not realizable.

Case 5. K: Absolute cone, $\Phi$ : Circle. The orthogonal projection of the vertex of $\mathbf{K}$ on $\Pi$ is the center of the circle $\Phi$. Hence, $\Pi$ is the only symmetry plane of $\Phi$ that does not contain the vertex of $\mathbf{K}$. Thus, only two camera positions are possible: the vertex of $\mathbf{K}$ and its reflection by $\Pi$. At both positions the camera may be oriented arbitrarily.

Case 6. K: Circular cone, $\Phi$ : Ellipse. The vertex of $\mathbf{K}$ must lie on the plane $\Pi_{\mathbf{1}}$, which is perpendicular to $\Pi$ and contains the major axis of $\Phi$. Combining reflections in symmetry planes of $\Phi$ leads to the total of 4 possible camera positions, forming a rectangle in $\Pi_{1}$. At each position, the camera can be oriented as discussed in section 4.2.4.

Case 7. K: Circular cone, $\Phi$ : Circle. As in Case 5, the orthogonal projection of the vertex of $\mathbf{K}$ on $\Pi$ is the center of $\Phi$, and there are only 2 possible camera positions. At each position, the camera may rotate arbitrarily about the line joining the projection centers or by $180^{\circ}$ about perpendicular lines. This is a sub-case of Case 5 .

Case 8. K: Elliptic cone, $\Phi$ : Ellipse. There are 8 possible camera positions which are the corners of a rectangular parallelepiped. At each position, 4 orientations are possible. This case is illustrated in Figure 1 (d).

Case 9. K: Elliptic cone, $\Phi$ : Circle. The only case where infi nitely many camera positions are possible: the camera may move on two circles (see section 4.2.3). At each position, 4 different orientations of the camera are possible.

Note that a $180^{\circ}$ rotation does not necessarily mean that the gaze directions before and after the rotation are opposite, e.g. if the rotation is about the optical axis, they even coincide.

\section{A Taxonomy of the CMS}

We now summarize the results of sections 4.1 and 4.2. Table 1 contains all classes of critical motion sequences, ordered by decreasing number of possible camera positions. The numbers of camera positions (\# $\mathbf{t}$ ) and orientations (\# $\mathbf{R}$ ) shown in the table, represent maximum confi gurations. \# $\Omega$, the number of potential absolute conics, indicates the lowest achievable degree of ambiguity in the Euclidean reconstruction.

From Defi nition 2 it follows that the motion sequences in Classes 2-5 are those critical for affi ne reconstruction. The sequences of Class 5, which are not contained in any of Classes 1-4, are the only CMS, that are not critical for self-calibration. 


\begin{tabular}{|l|l|c|c|c|}
\hline Class & Description & $\# \mathbf{t}$ & $\# \mathbf{~ R}$ & $\# \Omega$ \\
\hline \hline 1 & See 4.1 .2 & $\infty^{3}$ & $2 \times \infty$ & $\infty$ \\
\hline 2 & Cf. Case 9 & $2 \times \infty$ & 4 & $\infty^{2}$ \\
\hline 3 & Cf. Case 8 & 8 & 4 & 4 \\
\hline 4 & Cf. Case 6 & 4 & $2 \times \infty$ & 3 \\
\hline 5 & Cf. Case 5 & 2 & $\infty^{3}$ & 2 \\
\hline
\end{tabular}

Table 1. A taxonomy of the critical motion sequences. "\# $\mathbf{t}$ " is the number of possible camera positions and "\# $\mathbf{R}$ " the number of possible orientations of the camera at each position. "\# $\Omega$ ” means the minimum number of potential absolute conics (including $\Omega$ itself).

\subsection{Practically Important Cases}

The taxonomy can be further refi ned. This is mainly interesting to obtain a specifi c description of the CMS that might have practical signifi cance. In Table 2, we show the classes of CMS likely to be met in applications. Orbital motion (see also Figure 1 (a)), planar motion (Figure 1 (c)) and pure translation are particularly common imaging conditions for object modeling or navigation. Pure rotations are a good means of calibrating a camera, because of the simple algebra and easier feature matching due to the absence of occlusions [11].

\begin{tabular}{|l|l|c|c|c|}
\hline Class & Description & $\# \mathbf{t}$ & $\# \mathbf{~ R}$ & $\# \Omega$ \\
\hline \hline 1.1 & See 4.1.2 and Figure 1 (b) & $\infty^{3}$ & $\infty$ & $\infty$ \\
\hline 1.1 .1 & Planar motion, Figure 1 (c) & $\infty^{2}$ & $\infty$ & $\infty$ \\
\hline 1.4 & Pure translations & $\infty^{3}$ & 1 & $\infty^{5}$ \\
\hline 2.1 & Orbital motion, Figure 1 (a) & $2 \times \infty$ & 1 & $\infty^{2}$ \\
\hline 5.2 & Pure rotations & 1 & $\infty^{3}$ & $\infty^{3}$ \\
\hline
\end{tabular}

Table 2. Practically important classes of critical motion sequences. The numbering of classes does not reflect completely the hierarchy, since for example Class 1.1.1 is also a sub-class of Class 2.

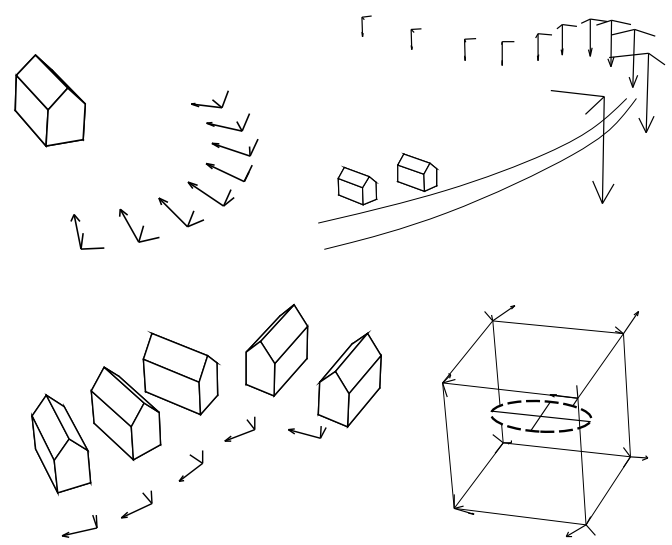

Figure 1. Illustration of critical motion sequences. The cameras are represented by the local coordinate axes, arcs indicate optical axes. (a) Orbital motion. (b) Rotation about parallel axes and arbitrary translation. (c) Planar motion. (d) A hypothetical critical sequence of Class 3. At each position, only one of the 4 possible orientations is shown.

\subsection{Degree of Ambiguity}

For the practically important CMS in Table 2, we indicate which level of reconstruction can be recovered. Sequences of Classes 1.1 and 1.1.1 permit Euclidean reconstruction up to a scale ambiguity in one direction [2,25]. Pure camera translations (Class 1.4) enable an affi ne reconstruction of the scene [17]. Of course, from pure rotations, no reconstruction at all is possible. From orbital motion (Class 2.1), the Euclidean structure of the planes parallel to the motion plane can be recovered, but there remains a two degree-of-freedom projective ambiguity perpendicular to it. 


\section{Practical Issues}

The problem of CMS, in contrast to critical surfaces ("Motion fi elds are hardly ever ambiguous" [14]) is important in practice, since many image sequences are actually critical.

Reducing Ambiguities. It is possible to reduce the degree of ambiguity in reconstructions, when prior knowledge of the scene structure or the camera motion is available. A known or partially known calibration of the camera should also be used if available: for applications in robotics, at least the stable camera parameters (aspect ratio and skew) are normally known. No fault is usually committed if the angle between the pixel axes is set to $90^{\circ}$. In [2], this constraint is used to reduce the ambiguity of Euclidean reconstruction from planar motion to a two-fold one. However, the constraint is added after having obtained a 1-degree ambiguous reconstruction, which shows that it is not always clear how to integrate constraints on the intrinsic parameters into a batch solution. Another way of discarding ambiguous solutions is to analyze reconstructions with respect to physical contradictions, e.g. to see whether a reconstructed point lies behind a camera by which it is actually seen. The best way to counter CMS is clearly to avoid them by using motion sequences that are "far" from critical: turning around an object while fi xating it is adequate for modeling, but critical if the camera is not calibrated; however, including rotations about the camera's optical axis turns the sequence into a non-critical one (photogrammetric experience has shown that rotations of $90^{\circ}$ produce good conditions for reconstruction).

Detecting and Identifying CMS. A general-purpose system for Euclidean reconstruction should at least be able to detect the presence of ambiguous solutions, e.g. by analyzing covariance matrices. Better would be the identifi cation of the type of the underlying motion sequence. This would enable the ambiguity in the reconstruction to be labeled correctly, e.g. as an affi ne reconstruction. Furthermore, if the CMS is identifi ed, a special algorithm might be applied to achieve a more stable and accurate reconstruction. A "brute force" approach would be to execute all available algorithms, designed for general or critical sequences. The adequate solution could then be determined by a model selection phase: the "smallest" model (reconstruction with lowest geometric level) which fi ts the data acceptably well (e.g. with a reprojection error below a threshold). An algorithm that identifi es singular motions in order to obtain less complex constraint equations for self-calibration than Kruppa's equations is described in [24]. However, only frameto-frame motions are considered, and thus critical motion sequences are not identifi ed and may cause the algorithm to fail.

\section{Conclusion}

We have derived all types of motion sequences for which monocular uncalibrated Euclidean reconstruction is ambiguous. By identifying several typical imaging conditions as critical we have shown that critical sequences are important in practice so that they should be taken into account when developing systems for Euclidean reconstruction. It is clear, that ambiguities can often be reduced right from the start by using an available partial calibration or just by avoiding critical motions.

Work in progress is concerned with the investigation of approaches for the implementation of a stratifi ed reconstruction system. We are also working on an extension of our formalism to the affi ne camera model and to the case of a zooming camera. A study similar to that of the "Gefährliche Räume" [13] would also be interesting, i.e. investigating how near a motion sequence must be to critical in order to cause inherent numerical instabilities.

Acknowledgements. I would like to thank Long Quan and Bill Triggs for fruitful discussions.

\section{References}

1. G. Adiv, "Inherent Ambiguities in Recovering 3-D Motion and Structure from a Noisy Flow Field", PAMI, (11) 5, 477-489, 1989.

2. M. Armstrong, A. Zisserman and R. Hartley, "Self-Calibration from Image Triplets", ECCV, Cambridge, UK, 3-16, 1996.

3. A. Basu, "Active Calibration: Alternative Strategy and Analysis", CVPR, New York, 495-500, 1993.

4. W. Boehm and H. Prautzsch, "Geometric Concepts for Geometric Design", A K Peters, 1994.

5. L. Dron, "Dynamic Camera Self-Calibration from Controlled Motion Sequences", CVPR, New York, 501-506, 1993. 
6. F. Du and M. Brady, "Self-Calibration of the Intrinsic Parameters of Cameras for Active Vision Systems", CVPR, New York, 477-482, 1993.

7. O. Faugeras, "What Can Be Seen in Three Dimensions with An Uncalibrated Stereo Rig ?", ECCV, Italy, 563-578, 1992.

8. O. Faugeras, "Stratifi cation of Three-Dimensional Vision: Projective, Affi ne and Metric Representations", Journal Opt. Soc. Am. A, (12), 465-484, 1995.

9. R. Hartley, R. Gupta and T. Chang, "Stereo from Uncalibrated Cameras", ECCV, Italy, 761-764, 1992.

10. R.I. Hartley, "Euclidean Reconstruction from Uncalibrated Views", Darpa-Esprit Worksh. Applications of Invariants in Computer Vision, Azores, Portugal, 187-202, 1993.

11. R.I. Hartley, "Self-Calibration from Multiple Views with a Rotating Camera", ECCV, Stockholm, 471-478, 1994.

12. A. Heyden and K. Åström, "Euclidean Reconstruction from Constant Intrinsic Parameters", ICPR, Vienna, 339343, 1996.

13. W. Hofmann, "Das Problem der Gefährlichen Flächen in Theorie und Praxis - Ein Beitrag zur Hauptaufgabe der Photogrammetrie", PhD Thesis, TU München, Germany, 1953.

14. B.K.P. Horn, "Motion Fields are Hardly Ever Ambiguous", IJCV, (1) 3, 259-274, 1987.

15. Q.-T. Luong, "Matrice fondamentale et autocalibration en vision par ordinateur", PhD Thesis, Paris-Orsay, France, 1992.

16. S.J. Maybank and O.D. Faugeras, "A Theory of Self Calibration of a Moving Camera", IJCV, (8) 2, 123-151, 1992.

17. T. Moons, L. Van Gool, M. Van Diest and E. Pauwels, "Affi ne Reconstruction from Perspective Image Pairs", Darpa-Esprit Worksh. Applications of Invariants in Computer Vision, Azores, Portugal, 249-266, 1993.

18. M. Pollefeys and L. Van Gool, "A Stratifi ed Approach to Metric Self-Calibration”, CVPR, Puerto Rico, 1997.

19. J.G. Semple and G.T. Kneebone, "Algebraic Projective Geometry", Oxford Science Publication, 1952.

20. C.C. Slama (Ed.), "Manual of Photogrammetry", 4th Edition, American Soc. of Photogrammetry and Remote Sensing, 1980.

21. P. Sturm, "Vision 3D non calibrée : contributions à la reconstruction projective et étude des mouvements critiques pour l'auto-calibrage", PhD Thesis, INPG, France, 1997.

22. R. Szeliski and S.B. Kang, "Shape Ambiguities in Structure from Motion", ECCV, Cambridge, UK, 709-721, 1996.

23. B. Triggs, "Autocalibration and the Absolute Quadric", CVPR, Puerto Rico, 1997.

24. T. Viéville and D. Lingrand, "Using Singular Displacements for Uncalibrated Monocular Visual Systems", ECCV, Cambridge, UK, 207-216, 1996.

25. C. Wiles and M. Brady, "Ground Plane Motion Camera Models", ECCV, Cambridge, UK, 238-247, 1996.

26. C. Zeller and O. Faugeras, "Camera Self-Calibration from Video Sequences: the Kruppa Equations Revisited", Research Report 2793, InRIA, France, 1996. 